\title{
Studies of multibody charmless B decays at LHCb
}

\author{
Fernando Luiz Ferreira Rodrigues ${ }^{* \dagger}$ \\ Centro Brasileiro De Pesquisas Físicas \\ E-mail: frodriguecbpf.br
}

During 2011, LHCb has collected an integrated luminosity of $1.0 \mathrm{fb}^{-1}$ giving rise to a large variety of measurements. The last results in multibody charmless $\mathrm{B}$ decays are presented: the evidence for $C P$ violation in $\mathrm{B}^{ \pm} \rightarrow \mathrm{K}^{ \pm} \pi^{+} \pi^{-}$and $\mathrm{B}^{ \pm} \rightarrow \mathrm{K}^{ \pm} \mathrm{K}^{+} \mathrm{K}^{-}$decays, that is the first evidence of inclusive $C P$ asymmetry in charmless three-body B decays, branching fraction measurements of $\mathrm{B}_{d, s}^{0} \rightarrow \mathrm{K}_{\mathrm{s}}^{0} h^{ \pm} h^{\prime \mp}$ decays and measurements of the polarization amplitudes, strong phase difference and triple product asymmetries in the $\mathrm{B}_{s}^{0} \rightarrow \phi \phi$ decay.

36th International Conference on High Energy Physics

4-11 July 2012

Melbourne, Australia

* Speaker.

† On behalf of the LHCb Collaboration. 


\section{1. $C P$ violation in $\mathrm{B}^{ \pm} \rightarrow \mathrm{K}^{ \pm} \pi^{+} \pi^{-}$and $\mathrm{B}^{ \pm} \rightarrow \mathrm{K}^{ \pm} \mathrm{K}^{+} \mathrm{K}^{-}$}

Charmless three-body decays give the possibility to study the weak phase in interference patterns between two-body resonances in the Dalitz plot. The measurement of the integrated charge asymmetry as well as the asymmetry distributions in the Dalitz plot for $\mathrm{B}^{ \pm} \rightarrow \mathrm{K}^{ \pm} \pi^{+} \pi^{-}$and $\mathrm{B}^{ \pm} \rightarrow \mathrm{K}^{ \pm} \mathrm{K}^{+} \mathrm{K}^{-}$decays, performed at $\mathrm{LHCb}$ using a sample of data corresponding to $1.0 \mathrm{fb}^{-1}$ are presented [1]. BaBar has claimed evidence of $C P$ violation in the final state $\mathrm{B}^{+} \rightarrow \phi(1020) \mathrm{K}^{+}$with $\phi(1020) \rightarrow \mathrm{K}^{+} \mathrm{K}^{-}$through an amplitude analysis [2] and claimed evidence of $C P$ violation in the $\mathrm{B}^{+} \rightarrow \rho^{0} \mathrm{~K}^{+}$with $\rho \rightarrow \pi^{+} \pi^{-}$[3].

The analysis selects events where there is at least one candidate with high transverse energy, to pass the hadron trigger at the hardware stage. The subsequent software trigger selects two-, three- or four-track secondary vertex with high transverse momenta and significant displacement from the primary vertex. Also, selection requirements on topological variables such as the flight distance of the B candidate or the direction of its momentum vector are used as the main discriminant information. In this way, strong cuts on the momenta of the daughter particles are avoid hence minimising the variation of the selection efficiency over the Dalitz plot. Then, an inclusive offline selection for the $\mathrm{B}^{ \pm} \rightarrow h^{ \pm} h^{+} h^{-}$decays, thanks to the similar topology, is applied.

The signal yields are determined from unbinned extended maximum likelihood fits (see Figure 1 for the $\mathrm{B}^{ \pm} \rightarrow \mathrm{K}^{ \pm} \pi^{+} \pi^{-}$and the $\mathrm{B}^{ \pm} \rightarrow \mathrm{K}^{ \pm} \mathrm{K}^{+} \mathrm{K}^{-}$mass fits). From the signal yields of $\mathrm{B}^{+}$and $\mathrm{B}^{-}$, a raw data asymmetry $\left(A_{C P}^{\mathrm{RAW}}\right)$ is calculated. In order to determine the $C P$ asymmetry $\left(A_{C P}\right)$, the effects of production and detection asymmetries need to be subtracted from the observed raw data asymmetry. This subtraction is performed by using the control channel $\mathrm{B}^{ \pm} \rightarrow \mathrm{J} / \psi \mathrm{K}^{ \pm}$corrected by its $C P$ asymmetry:

$$
A_{C P}\left(\mathrm{~B}^{ \pm} \rightarrow \mathrm{K}^{ \pm} h^{+} h^{-}\right)=A_{C P}^{\mathrm{RAW}}\left(\mathrm{B}^{ \pm} \rightarrow \mathrm{K}^{ \pm} h^{+} h^{-}\right)-A_{C P}^{\mathrm{RAW}}\left(\mathrm{B}^{ \pm} \rightarrow \mathrm{J} / \psi \mathrm{K}^{ \pm}\right)+A_{C P}\left(\mathrm{~B}^{ \pm} \rightarrow \mathrm{J} / \psi \mathrm{K}^{ \pm}\right)
$$

The asymmetry for $\mathrm{B}^{ \pm} \rightarrow \mathrm{K}^{ \pm} \pi^{+} \pi^{-}$and $\mathrm{B}^{ \pm} \rightarrow \mathrm{K}^{ \pm} \mathrm{K}^{+} \mathrm{K}^{-}$are

$$
\begin{aligned}
& A_{C P}\left(\mathrm{~B}^{ \pm} \rightarrow \mathrm{K}^{ \pm} \pi^{+} \pi^{-}\right)=+0.034 \pm 0.009(\text { stat }) \pm 0.004(\text { syst }) \pm 0.007(J / \psi K) \\
& A_{C P}\left(\mathrm{~B}^{ \pm} \rightarrow \mathrm{K}^{ \pm} \mathrm{K}^{+} \mathrm{K}^{-}\right)=-0.046 \pm 0.009(\text { stat }) \pm 0.005(\text { syst }) \pm 0.007(\mathrm{~J} / \psi K)
\end{aligned}
$$

These inclusive measurements have $2.8 \sigma$ and $3.7 \sigma$ significances for the $\mathrm{B}^{ \pm} \rightarrow \mathrm{K}^{ \pm} \pi^{+} \pi^{-}$and $\mathrm{B}^{ \pm} \rightarrow \mathrm{K}^{ \pm} \mathrm{K}^{+} \mathrm{K}^{-}$decays, respectively. Both measurements are in agreement with the current PDG values, that are $A_{C P}\left(\mathrm{~B}^{ \pm} \rightarrow \mathrm{K}^{ \pm} \pi^{+} \pi^{-}\right)=0.038 \pm 0.028$ [5] and $A_{C P}\left(\mathrm{~B}^{ \pm} \rightarrow \mathrm{K}^{ \pm} \mathrm{K}^{+} \mathrm{K}^{-}\right)=-0.017 \pm 0.030$ [5]. The result obtained for $\mathrm{B}^{ \pm} \rightarrow \mathrm{K}^{ \pm} \mathrm{K}^{+} \mathrm{K}^{-}$is the first evidence of inclusive $C P$ asymmetry in charmless three-body $\mathrm{B}^{ \pm}$decays.

In adition to the integrated charge asymmetry measurement, values of $A_{C P}^{\mathrm{RAW}}$ in bins of the two-body invariant mass projections have been determined. The signal yield in each bin has been estimated from a simplified invariant mass fit due to the limited statistics. In Figure 2 the $\mathrm{B}^{ \pm} \rightarrow \mathrm{K}^{ \pm} \mathrm{K}^{+} \mathrm{K}^{-}$projections using low two-kaon invariant mass defined as $m_{\mathrm{K}^{+} \mathrm{K}^{-} \text {low }}^{2}<m_{\mathrm{K}^{+} \mathrm{K}^{-} \text {high }}^{2}$ and $\mathrm{B}^{ \pm} \rightarrow \mathrm{K}^{ \pm} \pi^{+} \pi^{-}$projections using $m_{\pi^{+} \pi^{-}}^{2}$ are reported. These two projections present the highest $A_{C P}^{\mathrm{RAW}}$ region. We observed large opposite sign asymmetries in both invariant masses projections not clearly associated to a resonant state.

\section{Branching fraction measurements of $\mathrm{B}_{d, s}^{0} \rightarrow \mathrm{K}_{\mathrm{S}}^{0} h^{ \pm} h^{\prime \mp}$}

The study of the charmless three-body decays of neutral B mesons to final states including a $\mathrm{K}_{\mathrm{S}}^{0}$ meson, namely $\mathrm{B}_{d, s}^{0} \rightarrow \mathrm{K}_{\mathrm{s}}^{0} \pi^{ \pm} \pi^{\mp}, \mathrm{B}_{d, s}^{0} \rightarrow \mathrm{K}_{\mathrm{s}}^{0} \mathrm{~K}^{ \pm} \pi^{\mp}$ and $\mathrm{B}_{d, s}^{0} \rightarrow \mathrm{K}_{\mathrm{s}}^{0} \mathrm{~K}^{ \pm} \mathrm{K}^{\mp}$, has a number of physics goals. As first 

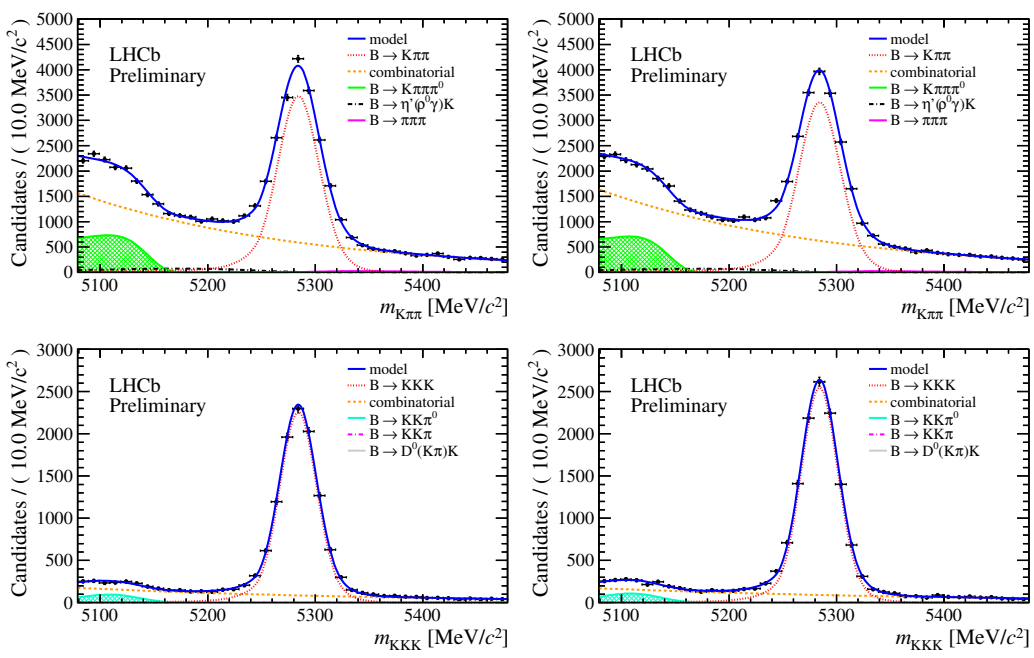

Figure 1: Fits to the invariant mass distributions of (first row) $\mathrm{B}^{ \pm} \rightarrow \mathrm{K}^{ \pm} \pi^{+} \pi^{-}$and (second row) $\mathrm{B}^{ \pm} \rightarrow \mathrm{K}^{ \pm} \mathrm{K}^{+} \mathrm{K}^{-}$ candidates for $B^{-}$(left) and $B^{+}$(right). The data are shown as points with error bars. Each component of the fit model is displayed on the plot.
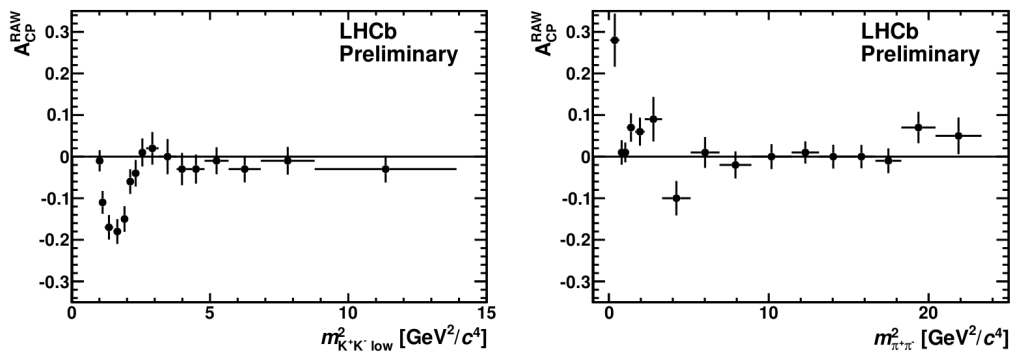

Figure 2: $A_{C P}^{\mathrm{RAW}}$ distributions for (left) $\mathrm{B}^{ \pm} \rightarrow \mathrm{K}^{ \pm} \mathrm{K}^{+} \mathrm{K}^{-}$Dalitz variable $m_{\mathrm{K}^{+} \mathrm{K}^{-} \text {low }}^{2}$ and (right) $\mathrm{B}^{ \pm} \rightarrow \mathrm{K}^{ \pm} \pi^{+} \pi^{-}$ Dalitz variable $m_{\pi^{+} \pi^{-}}^{2}$.

step towards to the physics results is to establish the signals in these modes. The branching fractions measurements of five $\mathrm{B}_{d, s}^{0} \rightarrow \mathrm{K}_{\mathrm{s}}^{0} h^{ \pm} h^{\prime \mp}$ decay modes relative to that of $\mathrm{B}^{0} \rightarrow \mathrm{K}_{\mathrm{s}}^{0} \pi^{ \pm} \pi^{\mp}$ are presented [6].

The analysis required a positive decision from the hadron trigger and applied topological variables cuts as in $\mathrm{B}^{ \pm} \rightarrow h^{ \pm} h^{+} h^{-}$decay modes selection. A multivariate discriminant based on a boosted decision tree has been designed in order to complete the final selection. Since two thirds of the reconstructed $\mathrm{K}_{\mathrm{s}}^{0}$ mesons decay downstream of the Vertex Locator, it is necessary, to divide the $\mathrm{K}_{\mathrm{s}}^{0}$ candidates into two categories: those whose daughter tracks do have Vertex Locator information in addition to information from the tracking stations, and those that have information only from the tracking stations, which we dub "Long-Long" and "Down-Down", respectively.

The signal yields are determined from a simultaneous unbinned extended maximum likelihood fit to the invariant mass distributions of all decays channels (see Figure 3 for the $K_{s}^{0} \pi^{ \pm} \pi^{\mp}$, the $K_{s}^{0} K^{ \pm} \pi^{\mp}$ and the $\mathrm{K}_{\mathrm{S}}^{0} \mathrm{~K}^{ \pm} \mathrm{K}^{\mp}$ spectra). The measurement of the five channels branching fractions relative to the 

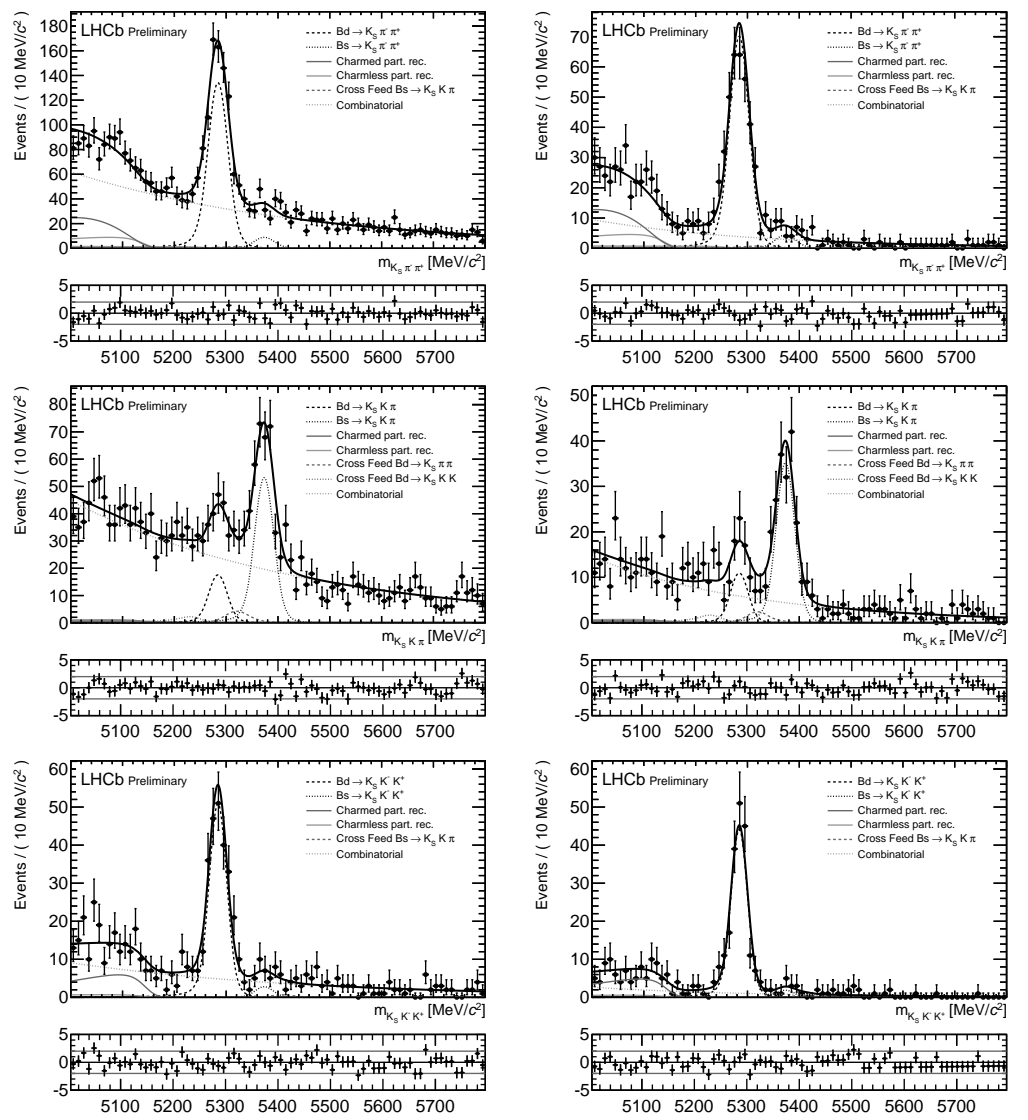

Figure 3: (first row) $\mathrm{K}_{\mathrm{S}}^{0} \pi^{ \pm} \pi^{\mp}$, (second row) $\mathrm{K}_{\mathrm{S}}^{0} \mathrm{~K}^{ \pm} \pi^{\mp}$ and (third row) $\mathrm{K}_{\mathrm{S}}^{0} \mathrm{~K}^{ \pm} \mathrm{K}^{\mp}$ spectra for $\mathrm{K}_{\mathrm{S}}^{0}$ reconstructed in the (left) Down-Down and (right) Long-Long categories. The data are described by the points. Each component of the fit model is displayed on the plot. The residuals of the fit with respect to data are shown on the bottom of the plots.

well-established branching fraction of the $\mathrm{B}^{0} \rightarrow \mathrm{K}_{\mathrm{S}}^{0} \pi^{ \pm} \pi^{\mp}$ from B-factories are

$$
\begin{aligned}
& \frac{\mathscr{B}\left(\mathrm{B}^{0} \rightarrow \mathrm{K}_{\mathrm{s}}^{0} \mathrm{~K}^{ \pm} \pi^{\mp}\right)}{\mathscr{B}\left(\mathrm{B}^{0} \rightarrow \mathrm{K}_{\mathrm{s}}^{0} \pi^{ \pm} \pi^{\mp}\right)}=0.117 \pm 0.018 \text { (stat) } \pm 0.018 \text { (syst) }, \\
& \frac{\mathscr{B}\left(\mathrm{B}^{0} \rightarrow \mathrm{K}_{\mathrm{s}}^{0} \mathrm{~K}^{ \pm} \mathrm{K}^{\mp}\right)}{\mathscr{B}\left(\mathrm{B}^{0} \rightarrow \mathrm{K}_{\mathrm{s}}^{0} \pi^{ \pm} \pi^{\mp}\right)}=0.53 \pm 0.04 \text { (stat) } \pm 0.04 \text { (syst), } \\
& \frac{\mathscr{B}\left(\mathrm{B}_{s}^{0} \rightarrow \mathrm{K}_{\mathrm{s}}^{0} \pi^{ \pm} \pi^{\mp}\right)}{\mathscr{B}\left(\mathrm{B}^{0} \rightarrow \mathrm{K}_{\mathrm{s}}^{0} \pi^{ \pm} \pi^{\mp}\right)}=0.24 \pm 0.06 \text { (stat) } \pm 0.04 \text { (syst), } \\
& \frac{\mathscr{B}\left(\mathrm{B}_{s}^{0} \rightarrow \mathrm{K}_{\mathrm{s}}^{0} \mathrm{~K}^{ \pm} \pi^{\mp}\right)}{\mathscr{B}\left(\mathrm{B}^{0} \rightarrow \mathrm{K}_{\mathrm{s}}^{0} \pi^{ \pm} \pi^{\mp}\right)}=1.96 \pm 0.15 \text { (stat) } \pm 0.20 \text { (syst), } \\
& \left.\frac{\mathscr{B}\left(\mathrm{B}_{s}^{0} \rightarrow \mathrm{K}_{\mathrm{s}}^{0} \mathrm{~K}^{ \pm} \mathrm{K}^{\mp}\right)}{\mathscr{B}\left(\mathrm{B}^{0} \rightarrow \mathrm{K}_{\mathrm{s}}^{0} \pi^{ \pm} \pi^{\mp}\right)}=0.084 \pm 0.031 \text { (stat }\right) \pm 0.019 \text { (syst) } .
\end{aligned}
$$

The observation of $\mathrm{B}^{0} \rightarrow \mathrm{K}_{\mathrm{S}}^{0} \mathrm{~K}^{ \pm} \pi^{\mp}$ by BaBar [7] has been confirmed, with a statistical significance of $7.3 \sigma$. First evidence has been obtained for the decay modes $\mathrm{B}_{s}^{0} \rightarrow \mathrm{K}_{\mathrm{s}}^{0} \pi^{ \pm} \pi^{\mp}$ and $\mathrm{B}_{s}^{0} \rightarrow \mathrm{K}_{\mathrm{s}}^{0} \mathrm{~K}^{ \pm} \mathrm{K}^{\mp}$ with 
statistical significances of $4.5 \sigma$ and $3.3 \sigma$ respectively. The decay $\mathrm{B}_{s}^{0} \rightarrow \mathrm{K}_{\mathrm{S}}^{0} \mathrm{~K}^{ \pm} \pi^{\mp}$ has been observed for the first time.

\section{3. $\mathrm{B}_{s}^{0} \rightarrow \phi \phi$ measurements}

Studies of the polarization amplitudes and triple product asymmetries in the $\mathrm{B}_{s}^{0} \rightarrow \phi \phi$ decay provide powerful tests for the presence of contributions from processes beyond the Standard Model. Since this decay is a pseudoscalar to vector-vector transition, there are three possible spin configurations of the vector meson pair, that are represented by three helicity states. The helicity amplitudes can be related to linear polarization amplitudes where the longitudinal $\left(A_{0}\right)$ and parallel $\left(A_{\|}\right)$amplitude components are $C P$-even eigenstate, the perpendicular amplitude componente $\left(A_{\perp}\right)$ is $C P$-odd eigenstate and the strong phase is defined as $\delta_{\|} \equiv \arg \left(A_{\|} / A_{0}\right)$. From the V-A structure of the weak interaction, the longitudinal component, $f_{L}=\left|A_{0}\right|^{2} /\left(\left|A_{0}\right|^{2}+\left|A_{\perp}\right|^{2}+\left|A_{\|}\right|^{2}\right)$, is expected to be dominant.

In addition, a search for physics beyond Standard Model is performed by studying the triple product asymmetries in the $\mathrm{B}_{s}^{0} \rightarrow \phi \phi$ decay. Non-zero values of these quantities can be either due to $T$-violation or final-state interactions. Considering a $\mathrm{B}_{s}^{0} \rightarrow \phi \phi$ decay, where the $\mathrm{K}^{+}$momentum in the $\phi_{1,2}$ rest frame, and the parent $\phi_{1,2}$ momentum in the rest frame of the $\mathrm{B}_{s}^{0}$ meson span the two $\phi$ meson decay planes, $\theta_{1,2}$ is the angle between the $\mathrm{K}^{+}$track momentum in the $\phi_{1,2}$ meson rest frame and the parent $\phi_{1,2}$ momentum in the rest $\mathrm{B}_{s}^{0}$ rest frame, $\Phi$ is the angle between the two $\phi$ meson decay planes and $\vec{n}_{1,2}$ is the unit vector normal to the decay plane of the $\phi_{1,2}$ meson. There are two triple products observables that can be described by the decay angles $\theta_{1,2}$ and unit vector normal $\vec{n}_{1,2}$. The triple products observables are $U=\sin (2 \Phi) / 2$ and $V= \pm \sin (\Phi)$, where the positive sign is taken if the $T$-even quantity $\cos \theta_{1} \cos \theta_{2} \geq 0$ and the negative sign otherwise. With these terms $U$ and $V$, the two triple product asymmetries $A_{U}$ and $A_{V}$ are defined as

$$
A_{U}=\frac{N_{+}-N_{-}}{N_{+}+N_{-}}, \quad \text { and } \quad A_{V}=\frac{M_{+}-M_{-}}{M_{+}+M_{-}}
$$

where $N_{+}\left(N_{-}\right)$and $M_{+}\left(M_{-}\right)$are the number of events with $U>0(U<0)$ and $V>0(V<0)$ respectively. The distributions of the $\mathrm{U}$ and $\mathrm{V}$ triple product observables are show in Figure 4.

The polarization amplitudes, strong phase difference and triple product asymmetries in the $\mathrm{B}_{s}^{0} \rightarrow \phi \phi$ decay mode are measured to be

$$
\begin{aligned}
\left|A_{0}\right|^{2} & =0.365 \pm 0.022(\text { stat }) \pm 0.012(\text { syst }) \\
\left|A_{\perp}\right|^{2} & =0.291 \pm 0.024(\text { stat }) \pm 0.010(\text { syst }) \\
\left|A_{\|}\right|^{2} & =0.344 \pm 0.024(\text { stat }) \pm 0.014(\text { syst }) \\
\cos \left(\delta_{\|}\right) & =-0.844 \pm 0.068(\text { stat }) \pm 0.029(\text { syst }) \\
A_{U} & =-0.055 \pm 0.036(\text { stat }) \pm 0.018(\text { syst }) \\
A_{V} & =0.010 \pm 0.036 \text { (stat }) \pm 0.018(\text { syst })
\end{aligned}
$$

Note that the value of $f_{L}$ found in the $\mathrm{B}_{s}^{0} \rightarrow \phi \phi$ channel is almost equal to that in the $B_{s}^{0} \rightarrow K^{* 0} \bar{K}^{* 0}$ decay $\left(f_{L}=0.31 \pm 0.12 \pm 0.04\right.$ [9]). These values agree well with the CDF measurements [10] and the $A_{U}$ and $A_{V}$ measurement are consistent with the hypothesis of $C P$ conservation. 

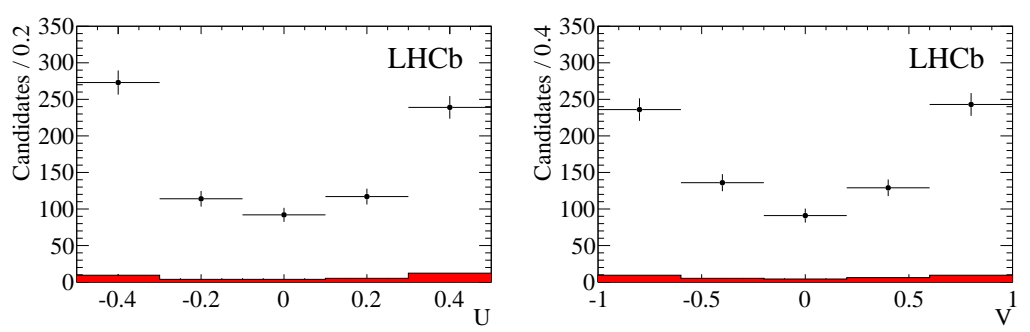

Figure 4: Distribution of the $\mathrm{U}$ (left) and $\mathrm{V}$ (right) observables for the $\mathrm{B}_{s}^{0} \rightarrow \phi \phi$ data in the mass range $5286.6 \mathrm{MeV} / c^{2}<M\left(\mathrm{~K}^{+} \mathrm{K}^{-} \mathrm{K}^{+} \mathrm{K}^{-}\right)<5446.6 \mathrm{MeV} / c^{2}$. The distribution for the background is taken from the mass sidebands, normalized to the same mass range and is shown by the solid histogram.

\section{References}

[1] LHCb Collaboration, Evidence of $C P$ violation in $\mathrm{B}^{ \pm} \rightarrow \mathrm{K}^{ \pm} \pi^{+} \pi^{-}$and $\mathrm{B}^{ \pm} \rightarrow \mathrm{K}^{ \pm} \mathrm{K}^{+} \mathrm{K}^{-}$decays, LHCb-CONF-2012-018.

[2] B. Aubert, et al., Dalitz plot analysis of the $\mathrm{B}^{ \pm} \rightarrow \mathrm{K}^{ \pm} \mathrm{K}^{+} \mathrm{K}^{-}$, Phys.Rev.D 74 (2006) 032003. arXiv:hep-ex/0605003, doi:10.1103/PhysRevD.74.032003.

[3] B. Aubert, et. al., Evidence for direct $C P$ violation from Dalitz plot analysis of $\mathrm{B}^{ \pm} \rightarrow \mathrm{K}^{ \pm} \pi^{+} \pi^{-}$, Phys.Rev.D 78 (2008) 012004. arXiv:0803.4451, doi:10.1103/PhysRevD.78.012004.

[4] A. Garmash, et al., Evidence for large direct $C P$ violation in $\mathrm{B}^{ \pm} \rightarrow \rho(770)^{0} \mathrm{~K}^{ \pm}$from analysis of the three-body charmless $\mathrm{B}^{ \pm} \rightarrow \mathrm{K}^{ \pm} \pi^{+} \pi^{-}$decay, Phys.Rev.Lett. 96 (2006) 251803. arXiv:hep-ex/0512066, doi:10.1103/PhysRevLett.96.251803.

[5] J. Beringer, et al., Review of Particle Physics (RPP), Phys.Rev.D 86 (2012) 010001. doi:10.1103/PhysRevD.86.010001.

[6] LHCb Collaboration, Branching fraction measurements of $\mathrm{B}_{d, s}^{0}$ decays to $K S h^{ \pm} h^{\prime \mp}$ final states, including first observation of $\mathrm{K}_{\mathrm{S}}^{0} \mathrm{~K}^{ \pm} \pi^{\mp}$, LHCb-CONF-2012-023.

[7] BaBar collaboration, P. del Amo Sanchez et al., Observation of the rare decay $\mathrm{B}^{0} \rightarrow \mathrm{K}_{\mathrm{S}}^{0} \mathrm{~K}^{ \pm} \pi^{\mp}$, Phys.Rev.D 82 (2010) 031101, [arXiv:1003.0640]

[8] LHCb Collaboration, R. Aaij et al., Phys.Lett. B 713 (2012) 369-377

[9] LHCb Collaboration, R. Aaij, et al., Phys. Lett. B 709 (2012) 50, arXiv:1111.4183.

[10] CDF Collaboration, T. Aaltonen, et al., Phys.Rev.Lett. 107 (2011) 261802, arXiv:1107.4999. 